\title{
A multi channel coupling based approach for the prediction of the channel capacity of MIMO-systems
}

\author{
F. Hagebölling and U. Zölzer
}

Department of Signal Processing and Communications, Helmut Schmidt University - University of the Federal Armed Forces Hamburg, Holstenhofweg 85, 22043 Hamburg, Germany

\begin{abstract}
When installing Multiple Input Multiple Output (MIMO)-systems, the antenna positioning has a major influence upon the achievable transmission quality. To determine those antenna positions, which maximize the transmission quality, in adequate time, a computer based prediction of the channel capacity is imperative.

In this paper, we will show that Ray Tracing, which is a very popular prediction method and well suited for the prediction of transfer functions or power delay profiles, produces unacceptable errors when predicting the channel capacity of MIMO-systems. Furthermore we identify the source of the prediction errors and present a new algorithm, based on an approach known as Multi Channel Coupling (MCC), which avoids this error source.

Finally a comparison of the prediction results of our algorithm with prediction results gained with an Image Ray Tracer as well as with measured results is used to show the formidable increasement of prediction accuracy which can be gained by using our algorithm.
\end{abstract}

\section{Introduction}

In Foschini and Gans (1998) it was shown, that MIMOsystems using spatial multiplexing increase the channel capacity to such an extent, that an immensely higher spectral efficiency becomes possible.

When installing such MIMO-systems the count of positions, where antennas can be placed, is usually higher than the number of antennas, which are to be placed. Being aware of the fact, that the antenna positioning has a big impact on the spatial multiplexing capabilities of the MIMO-system and thereby the achievable transmission quality, a lot of effort

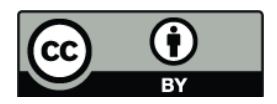

Correspondence to: F. Hagebölling (falko.hageboelling@hsu-hh.de) has already been taken to find antenna positionings which maximize transmission quality.

A lot publications concentrate on measuring channel capacities for different positionings in certain scenarios, Tang and Mohan (2005) and Fügen et al. (2002) shall be mentioned exemplarily. But measuring based approaches are always limited by the fact, that the number of possible MIMOsystems increases very fast with the number of possible antenna positions.

To be able to compare several thousand or more possible systems, computer based simulation is needed. In Talbi (2001) and Ziri-Castro et al. (2005) and a lot of other publications the received power was predicted and compared with measurements.

Though the results presented in those publications accord very well with measurements, the received power is, in our opinion, not a sufficient quantity to judge the transmission quality of MIMO-systems, because it doesn't contain any information about the linear independence of the Multiple Input Single Output (MISO)-systems contained in each MIMO-system. The latter is important, because in a spatial multiplexing MIMO-system the received signals can only be decoded properly, if the MISO-systems are linearly independent.

Though the channel capacity contains this important information only a comparatively small amount of publications deals with the prediction of this quantity. And most of the publications, which do so, don't present measurements as comparison for the prediction results. Elnaggar et al. (2004) may be named as example.

In the following we will briefly summarize some fundamentals in Sect. 2, compare results of a ray tracing based prediction algorithm with measurements and point out the disadvantages of this approach in Sect. 3, present our new algorithm in Sect. 4 and verify the better performace of our algorithm with more measurements in Sect. 5. A summary and conclusion marks can be found in Sect. 6 . 


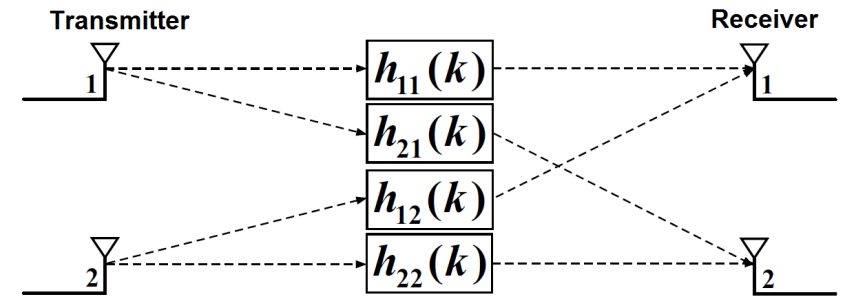

Fig. 1. SISO impulse responses of a MIMO Channel, $n_{\mathrm{T}}=n_{\mathrm{R}}=2$

\section{Fundamentals}

\subsection{Channel description}

The MIMO Channel with $n_{\mathrm{T}}$ transmit antennas and $n_{\mathrm{R}}$ receive antennas consists of one complex Single Input Single Output (SISO) impulse response between every transmit antenna $m$ and every receive antenna $n$ (see Fig. 1).

Assuming the same order $L$ for each of these impulse responses $\boldsymbol{h}_{n, m}=\left[h_{n, m}(0) h_{n, m}(1) \ldots h_{n, m}\left(L_{n, m}\right)\right]^{T}$ the frequency selective MIMO channel can be described by $L+1$ complex channel matrices $\mathbf{H}(k)$ with $k=0, \ldots, L$ :

$\mathbf{H}(k)=\left[\begin{array}{ccc}h_{1,1}(k) & \cdots & h_{1, n_{T}}(k) \\ \vdots & \ddots & \vdots \\ h_{n_{R}, 1}(k) & \cdots & h_{n_{R}, n_{T}}(k)\end{array}\right]$.

\subsection{Channel capacity}

Assuming that no power allocation strategy is used, the transmitted power $P_{\mathrm{T}}$ is uniformly distributed over the bandwidth $B$ and the Channel Capacity of a frequency selective MIMO system can be written as (Palomar et al., 2000)

$C=\int_{-B / 2}^{+B / 2} \log _{2}\left(\operatorname{det}\left[\mathbf{I}_{m}+\frac{P_{\mathrm{T}} /\left(n_{\mathrm{T}} \cdot B\right)}{\mathbf{S}_{n n}(f)} \mathbf{Q}(f)\right]\right) d f$,

with $S_{n n}(f)$ being the power spectral density matrix of the noise, $m=\min \left(n_{\mathrm{T}}, n_{\mathrm{R}}\right)$ and

$\mathbf{Q}(f)=\left\{\begin{array}{ll}\mathbf{H}_{\mathbf{F}}(f) \mathbf{H}_{\mathbf{F}}(f)^{H}, & n_{\mathrm{R}}<n_{\mathrm{T}} \\ \mathbf{H}_{\mathbf{F}}(f)^{H} \mathbf{H}_{\mathbf{F}}(f), & n_{\mathrm{R}} \geq n_{\mathrm{T}}\end{array}\right.$.

Discretizing this equation, assuming additive white noise and normalizing channel energy according to Bauch and AlDhahir (2002)

$\sum_{k=0}^{L} E\left\{\left|h_{n, m}(k)\right|^{2}\right\}=1 \quad \forall 1 \leq m \leq n_{\mathrm{T}}, 1 \leq n \leq n_{\mathrm{R}}$

leads to the formula

$C=\frac{1}{N_{\mathrm{F}}} \sum_{f=1}^{N_{\mathrm{F}}} \log _{2}\left(\operatorname{det}\left[\mathbf{I}_{m}+\frac{\rho}{n_{\mathrm{T}}} \mathbf{Q}(f)\right]\right)$,

with $N_{\mathrm{F}}$ being the number of discrete frequencies and $\rho$ being the mean Signal to Noise Ratio (SNR).

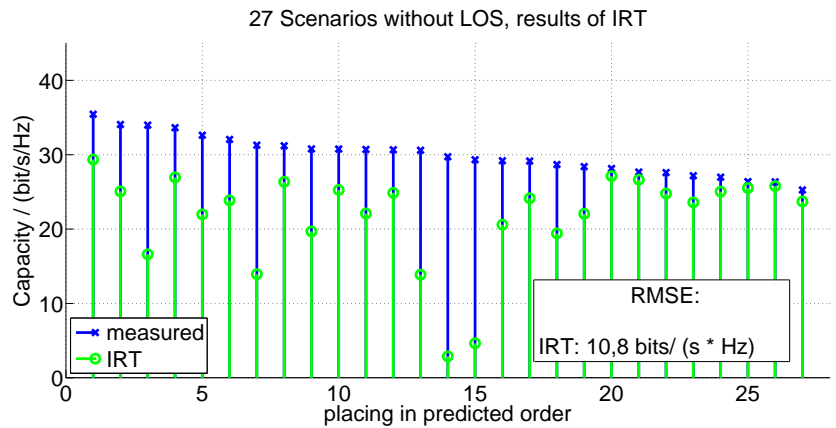

Fig. 2. Prediction results gained with image ray tracing.

The normalization according to Eq. (4) is needed to replace the term $\frac{P_{\mathrm{T}} /\left(n_{\mathrm{T}} \cdot B\right)}{\mathbf{S}_{n n}(f)}$ by $\rho$, but it abolishes differences in the path loss of the SISO-channels and reduces the comparability of different MIMO systems. To maintain the differences and to enable a comparison of $N_{M}$ MIMO systems the condition has to be relaxed to be (Hagebölling et al., 2006)

$\sum_{i=1}^{N_{M}} \sum_{m=1}^{n_{\mathrm{T}}} \sum_{n=1}^{n_{\mathrm{R}}} \sum_{k=0}^{L} E\left\{\left|h_{(n, m)_{i}}(k)\right|^{2}\right\}=N_{M} \cdot n_{\mathrm{T}} \cdot n_{\mathrm{R}}$.

\section{Image Ray Tracing}

In Hagebölling et al. (2006) we presented a prediction algorithm based on the very popular method of Image Ray Tracing (IRT). The algorithm determines all possible paths between each pair of transmit and receive antennas in a given scenario and with a given number of maximal reflections per path. It then identifies the points, where rays following these paths interact with the surrounding and calculates the impact of these interactions upon the electromagnetic field. We showed that the prediction of the channel capacity using this algorithm is in general very accurate but produces certain discrepancies for some scenarios.

Figure 2 shows some actual predictions of this algorithm for 27 indoor scenarios. The measurement of the channel capacity of this scenarios has been done using our laboritory MIMO system presented in Weikert and Zölzer (2005). In all scenarios there are $n_{\mathrm{T}}=4$ transmit antennas and $n_{\mathrm{R}}=4$ receive antennas, the carrier frequency is $2.49 \mathrm{GHz}$ and the SNR amounts $30 \mathrm{~dB}$.

Especially for the systems number 4, 5, 13, 14 and 15 the prediction error is very high.

In order to find the reason for the erroneous prediction of this scenarios, we prospected the propagation paths, which were used during the Ray Tracing process and those which were not used due to a too high number of reflections. It turned out that in all of this scenarios there are paths with a very low damping but with a very high number of reflections, mostly at metallic materials. 
Due to their low damping those paths imperatively have to be considered when calculating the impulse response, but because of their high number of reflections they were not considered by the algorithm. This error source is inherent for ray tracing based algorithms and can only be bated by increasing the maximum number of reflections per path.

However this measure does not solve the problem generally and is very expensive in terms of computation time and needed memory, because the complexity of Ray Tracing is of the order $w^{R}$ for $w$ walls and a maximal number of reflections per path $R$.

\section{Multi Channel Coupling}

Multi Channel Coupling (MCC) is a prediction algorithm, which takes an infinite number of interactions per path into account. It thereby avoids the mentioned error source, which is inherent for Ray Tracing based algorithms. MCC was first presented as algorithm to predict the transmission of power in 2-D-scenarios in Liebendorfer and Dersch (1997). This algorithm is based upon the following considerations:

- For every pair of walls there are two channels, one in each direction.

- Transmit antennas couple a certain percentage of their transmitted power into each channel.

- Each channel couples a certain percentage of the present power into each other channel.

- Each channel couples a certain percentage of the present power into each receive antenna.

Each coupling of transmit antennas into channels includes one reflection at or transmission through the start wall of the channel and each coupling from a channel into another channel is a reflection or transmission. Every coupling is described by a coupling factor between 0 and 1 and the coupling factors are organized in matrices: $\mathrm{a}\left(n_{\mathrm{C}} \times n_{\mathrm{T}}\right)$ matrix $\mathbf{T}$ for the coupling factors of the $n_{\mathrm{T}}$ transmit antennas into the $n_{\mathrm{C}}$ channels, a $\left(n_{\mathrm{C}} \times n_{\mathrm{C}}\right)$ matrix $\mathbf{C}$ for the coupling factors of channels into each other and a $\left(n_{\mathrm{R}} \times n_{\mathrm{C}}\right)$ matrix $\mathbf{R}$ for the coupling factors of the channels into the $n_{\mathrm{R}}$ receive antennas.

Assuming no direct component and considering an infinite number of couplings from channels into channels, i.e. an infinite count of reflections and transmissions, the power transfer matrix from the transmit antennas to the receive antennas can then be calulated by the equation

$$
\begin{aligned}
\mathbf{P} & =\mathbf{R} \cdot\left(\mathbf{T}+\mathbf{C} \mathbf{T}+\mathbf{C}^{2} \mathbf{T}+\cdots+\mathbf{C}^{\infty} \mathbf{T}\right) \\
& =\mathbf{R} \cdot \sum_{i=0}^{\infty} \mathbf{C}^{i} \mathbf{T}=\mathbf{R} \cdot(\mathbf{I}-\mathbf{C})^{-1} \mathbf{T}
\end{aligned}
$$

Thus MCC considers an infinite number of reflections and transmissions with the computation time being finite. Moreover the most computation time is needed for the calculation

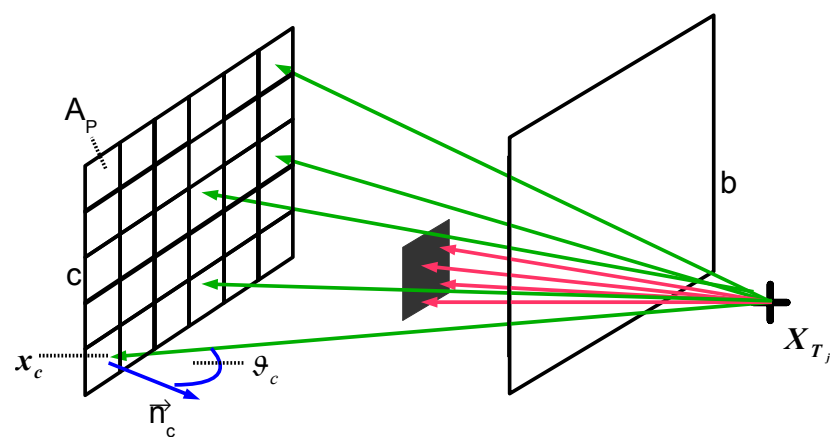

Fig. 3. Coupling from antenna $j$ into channel $i$; green rays contribute to the coupling factor.

of the matrix $(\mathbf{I}-\mathbf{C})^{-1}$, which only depends on the location and the material parameters of walls in the surrounding. Thus MCC is very effective, when calculating the same scenario several times with different antenna positions.

It was already shown, that the MCC method can also be used to predict other values than power. In Karthaus (2001) it was used to predict power delay profiles in 3-D-scenarios.

To be able to predict the channel capacity of frequency selective MIMO systems with and without line of sight using the MCC method, we write Eq. (7) as

$\mathbf{H}(f)=\mathbf{R}(f) \cdot(\mathbf{I}-\mathbf{C}(f))^{-1} \mathbf{T}(f)+\mathbf{D}(f)$

with $f$ being the frequency, $\mathbf{H}(f)$ being the complex transfer function in the frequency domain and the $\left(n_{\mathrm{R}} \times n_{\mathrm{T}}\right)$ matrix $\mathbf{D}(f)$ containing the coupling factors of the direct paths between each pair of transmit and receive antennas.

In the following we will present our formulas for the prediction of the channel capacity with Eq. 8.

\subsection{The elements of the matrix $T(f)$}

The element $T_{i j}$ describes, how an impulse at the transmit antenna $j$ is coupled into channel $i$.

To calculate $T_{i j}$, the end wall of channel $i$ is discretized. Then for each ray $p$ from the transmit antenna $T_{j}$ through one of the discrete wallelements with the area $A_{\mathrm{c}}$ it is calculated, how the amplitude and the phase of an impulse transmitted by the antenna is altered by the coupling.

Only rays, which intersect with the start wall of the channel and are not disturbed by other walls can contribute to the coupling factor. The impact on the amplitude is represented by $\tau_{p}$ and calculated using the portion of the solid angle, under which the wallelement is seen from the position of the transmit antenna, the antenna gain $G_{j}$ and the absolute directional characteristic $\left|C_{j, p}\right|$ of the antenna at path $p$ and the transmission / reflection coefficient $r_{p}(f)$ at the start wall of the channel. The superposition of all rays yields the absolute value of the coupling coefficient.

The impact on the phase is the sum of the phase of the transmission / reflection coefficient $r_{p}(f)$ and the phase shift 


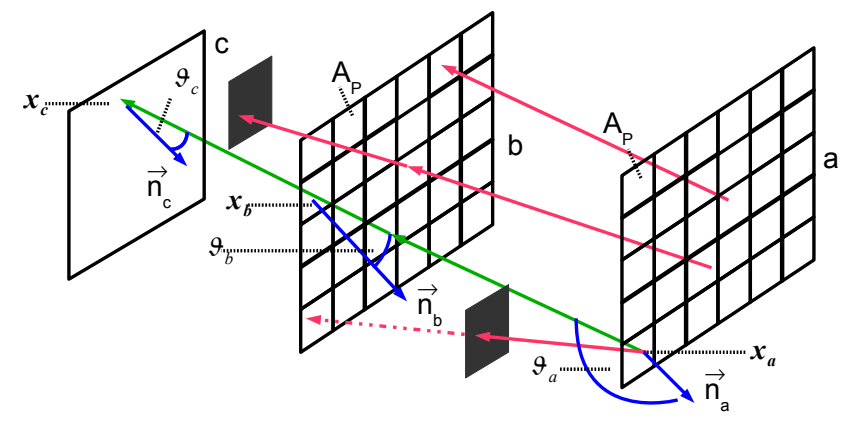

Fig. 4. Coupling from channel $j$ into channel $i$; green rays contribute to the coupling factor.

$\varphi_{0}$ caused by the free space propagation from the antenna to the wallelement. The phase of each ray $p$ is weighted with the according value $\tau_{p}$ and the mean value of all weighted phases is the phase of the coupling coefficient:

$T_{i j}(f)=\sqrt{\sum_{p} \tau_{p}(f)} \cdot e^{j \varphi(f)}$

with

$$
\begin{aligned}
& \tau_{p}(f)=\Delta_{1} \frac{A_{\mathrm{c}} \cdot \cos \left(\vartheta_{\mathrm{c}}\right)\left|x_{T_{j}}^{\prime}-x_{\mathrm{C}}\right|^{-2}}{4 \pi} \cdot G_{j}\left|C_{j, p}\right|\left|r_{p}(f)\right| \\
& \varphi(f)=\frac{\sum_{p}\left(\arg \left(r_{p}(f)\right)+\varphi_{0_{p}}(f)\right) \cdot \tau_{p}(f)}{\sum_{p} \tau_{p}}
\end{aligned}
$$

$\Delta_{1}=\left\{\begin{array}{l}1 \text { if ray from } T_{j} \text { through } A_{\mathrm{c}} \text { and } b \\ \text { is not interrupted } \\ 0 \text { else }\end{array}\right.$

\subsection{The elements of the matrix $\mathrm{C}(f)$}

The element $C_{i j}$ describes the coupling from channel $j$ into channel $i$ and is the expectation value of the ratio between the transfer function in channel $i$ and that one in channel $j$.

For the calculation, the start and the end wall of channel $j$ are discretized. Of all rays $p_{\alpha}$ from a discrete wall element of the start wall $a$ of channel $j$ through a discrete wall element $\alpha$ of the end wall $b$ of channel $j$, only those, which hit the end wall of channel $i$ and are not disturbed by other walls, contribute to the coupling. Again the absolute value of the coupling factor is obtained by the superposition of all contributing rays and its phase is the average of the weighted phases of the rays.

The wall element at wall $a$ has the area $A_{a}$ and is thought of as full radiator, which transmits in every direction proportional to $A_{a} \cos \left(\vartheta_{a}\right)$. The remaining, the coupling coeficcient defining quantities are the portion of the solid angle, under which the wallelement of wall $b$ is seen from the position $x_{a}$ and the transmission / reflection coefficient $r_{p}(f)$ at wall $b$. For the calculation of all transmission or reflexion coefficients the wave matrix method (Layer, 2001) is used, which allows the definition of walls with layers of different materials.

$C_{i j}(f)=\sqrt{\frac{\sum_{\alpha} \sum_{p_{\alpha}} X_{p_{\alpha}}}{\sum_{\alpha} \sum_{p_{\alpha}} \chi_{p_{\alpha}}(f)}} \cdot e^{j \varphi(f)}$

with

$\chi_{p}(f)=\Delta_{1} A_{a} \cos \left(\vartheta_{a}\right) A_{b} \cos \left(\vartheta_{b}\right)\left|\boldsymbol{x}_{a}-\boldsymbol{x}_{b}\right|^{-2}$

$X_{p} \quad=\Delta_{2} \chi_{p}(f)\left|r_{p}\right|$

$\varphi(f)=\frac{\sum_{\alpha} \sum_{p_{\alpha}}\left(\arg \left(r_{p_{\alpha}}(f)\right)+\varphi_{p_{p}}(f)\right) \cdot \Delta_{2} \chi_{p_{\alpha}}(f)\left|r_{p_{\alpha}}(f)\right|}{\sum_{\alpha} \sum_{p_{\alpha}} \Delta_{2} \chi_{p_{\alpha}}(f) r_{p_{\alpha}}(f)}$

$\Delta_{1}=\left\{\begin{array}{l}1 \text { if line from } A_{a} \text { to } A_{b} \\ \text { is not interrupted } \\ 0 \text { else }\end{array}\right.$

$\Delta_{2}=\left\{\begin{array}{l}1 \text { if ray from } A_{a} \text { through } c \\ \text { is not interrupted } \\ 0 \text { else }\end{array}\right.$

If the points $\boldsymbol{x}_{b}$ and $\boldsymbol{x}_{a}$ are equal, which is possible, if the walls $a$ and $b$ do intersect each other, only the portion of the solid angle, under which the wallelement of wall $c$ is seen from the position $x_{a}$ and the number $N_{c}$ of rays starting at $\boldsymbol{x}_{a}$, which hit wall $c$ are used to calculate the coupling factor. $\chi_{p}$ then is $\chi_{p}=1$ and

$X_{p}=\frac{1}{N_{c}} \sum_{p} \Delta_{2} \frac{A_{c} \cos \left(\vartheta_{c}\right)\left|\boldsymbol{x}_{a}-\boldsymbol{x}_{c}\right|^{-2}}{4 \pi}$.

\subsection{The elements of the matrix $R(f)$}

The element $R_{i j}$ describes how the signal is coupled from channel $j$ into the receive antenna $i$. The coupling factor is unequal to zero for all non disturbed rays $P_{\alpha}$ from a discrete wall element of the start wall $a$ of channel $j$ through the position of the receive antenna and a discrete wall element $\alpha$ of the end wall $b$ of channel $j$. Every ray is thought of as representing a subchannel of channel $j$, having the cross section area $A_{a}$ at wall $a, A_{\mathrm{R}}$ at the receive antenna and $A_{b}$ at wall $b$. The discrete wall element at wall $a$ is again thought of as full radiator. The coupling factor is further determined by the portion of the solid angle, under which the wallelement of wall $b$ is seen from the position $x_{a}$ and the ratio between the cross section area $A_{\mathrm{R}}$ and the aperture of receive antenna $\frac{G_{i}\left|C_{i, p}\right| \lambda^{2}}{4 \pi}$, where $G_{i}$ ist the antenna gain and $C_{i, p}$ is the directional characteristic of antenna $i$ at path $p$. 


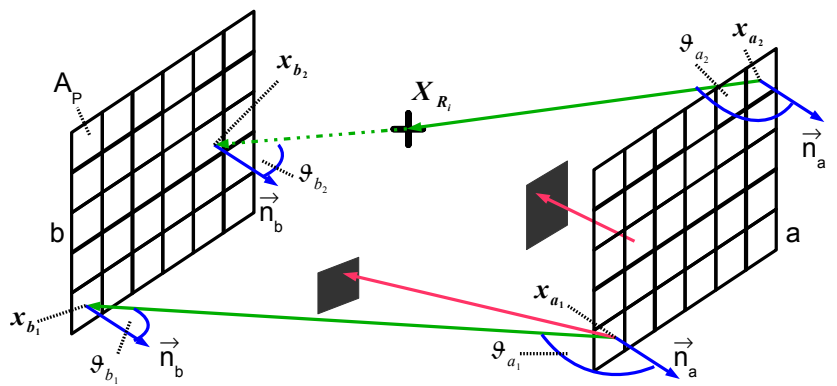

Fig. 5. Coupling from channel $j$ into antenna $i$; green rays contribute to the coupling factor.

$R_{i j}(f)=\sqrt{\frac{\sum_{\alpha} \sum_{p_{\alpha}} \Delta_{1} G_{i}\left|C_{i, p}\right| \frac{\lambda^{2}}{4 \pi A_{R}} \rho_{\alpha}}{\sum_{\alpha} \sum_{p_{\alpha}} \Delta_{2} \rho_{\alpha}}} \cdot e^{j \varphi(f)}$

with

$\rho(f)=A_{a} \cos \left(\vartheta_{a}\right) A_{b} \cos \left(\vartheta_{b}\right)\left|\boldsymbol{x}_{a}-\boldsymbol{x}_{b}\right|^{-2}$

$\varphi(f)=\sum_{\alpha} \frac{\varphi_{p_{p_{\alpha}}}(f) \rho_{p_{\alpha}}(f)}{\sum_{\alpha} \rho_{p_{\alpha}}(f)}$

$\Delta_{1}=\left\{\begin{array}{l}1 \text { if ray from } A_{a} \text { through } x_{R_{i}} \text { hits } A_{b} \\ \quad \text { without interruption } \\ 0 \text { else }\end{array}\right.$

$\Delta_{2}=\left\{\begin{array}{l}1 \text { if ray from } A_{a} \text { hits } A_{b} \\ \quad \text { without interruption } \\ 0 \text { else }\end{array}\right.$

Using the intercept theorem one can show that

$A_{\mathrm{R}}=4\left(\frac{\left|\boldsymbol{x}_{b}-\boldsymbol{x}_{R}\right|}{\left|\boldsymbol{x}_{a}-\boldsymbol{x}_{b}\right|}\right)^{2} A_{a} \cos \left(\vartheta_{a}\right)$

and that leads to a formula for $R_{i} j$ which needs only one summation over all rays $r$ from the discrete wall elements of wall $a$ to the receive antenna:

$R_{i j}(f)=\sqrt{\frac{\sum_{r} \kappa_{r}}{\sum_{\alpha} \sum_{p_{\alpha}} \Delta_{2} \rho_{\alpha}}} \cdot e^{j \varphi(f)}$

with

$\kappa(f)=\Delta_{1} \frac{\lambda^{2}}{16 \pi} G_{i}\left|C_{i, p}\right| \cos \left(\vartheta_{a}\right)\left|\boldsymbol{x}_{a}-\boldsymbol{x}_{B S}\right|^{-2}$

$\varphi(f)=\sum_{r} \frac{\varphi_{0_{r}}(f) \kappa_{r}(f)}{\sum_{r} \kappa_{r}(f)}$

and $\rho, \Delta_{1}$ and $\Delta_{2}$ remaining the same as in Eq. (15).

\subsection{The elements of the matrix $D(f)$}

The element $D_{i j}$ describes the free space distribution between transmit antenna $j$ and receive antenna $i$. It is calculated as

$D_{i j}(f)=\frac{r_{0}}{r} \cdot e^{-j \frac{2 \pi}{\lambda}\left(r-r_{0}\right)}$

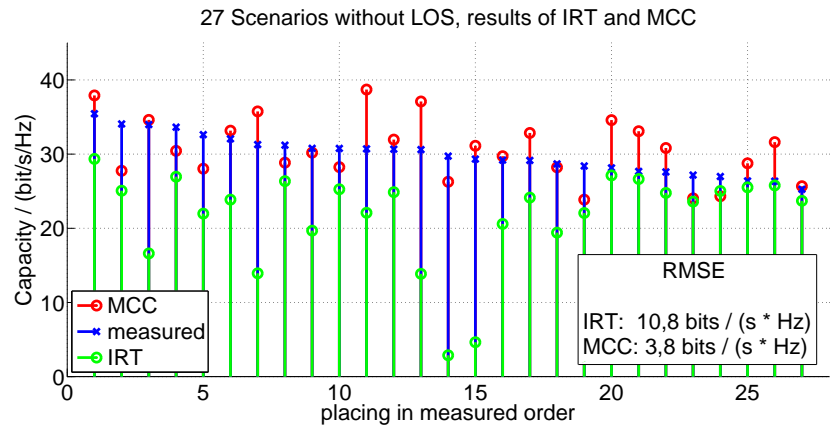

Fig. 6. Comparison of MCC and IRT.

with $r$ being the distance between the antennas and $r_{0}=$ $\lambda(4 \pi)^{-1}$. If the first fresnel zone is not free of obstacles, $D_{i j}$ is proportional to $r^{-2}$ instead of $r^{-1}$, which is known as double regression model.

\section{Appraisal of prediction results}

Figure 6 shows the results of the prediction of the 27 scenarios mentioned in Sect. 3 using the new algorithm. For comparison with the results of the Ray Tracing based algorithm and the measurements, this data is also shown in the figure. One can see, that the prediction using MCC does not produce the same big errors as that using IRT.

By avoiding the errors, which are inherent to measures, which can only handle a finite count of interactions, Multi Channel Coupling outperforms Image Ray Tracing in terms of the root mean square error (RMSE). While the RMSE of the Ray Tracing based prediction amounts up to 10.8 bits $(\mathrm{Hz} \mathrm{s})^{-1}$, MCC has a RMSE of only 3.8 bits $(\mathrm{Hz} \mathrm{s})^{-1}$, which is approximately a third of the first one. To further support the thesis above, Fig. 5 shows the absolute deviations of MCC and IRT from measurements for 108 indoor scenarios, 54 with line of sight (LOS) and 54 without one. In a scenario with a line of sight, usually the LOS is the path, where the most power is transfered to the receiver. Concomitantly, the LOS is always taken into recognition while the Ray Tracing based prediction, because the number of reflections on that path is zero.

Because of that and as the Ray Tracing based approach fails only when paths with a lot of interactions transfer a big part of the received power, one would expect the Ray Tracing based approach, to perform as good as MCC when the predicted scenario contains a line of sight. The results of the 54 systems with LOS confirm this expectation and that of the 54 systems without LOS show again, that MCC avoids the big errors, which cannot be avoided using Ray Tracing. 

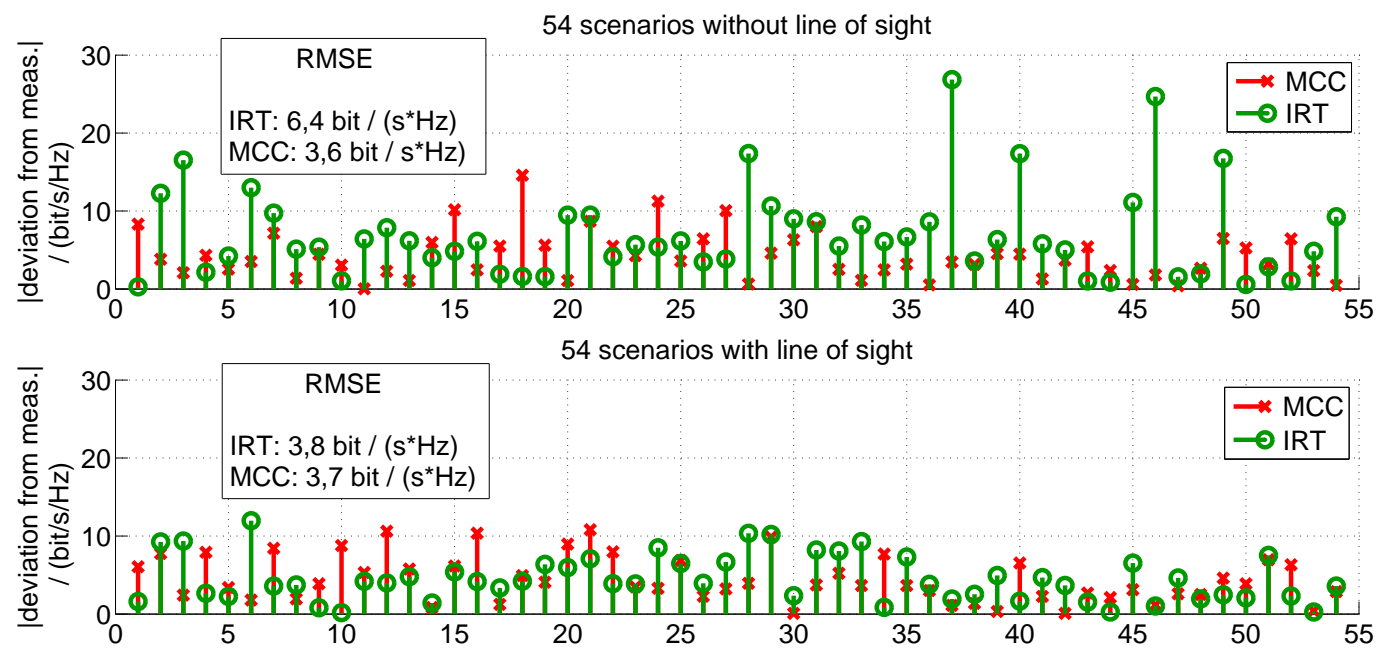

Fig. 7. Comparison of MCC and IRT: 108 scenarios.

\section{Conclusions}

We explained, why in our opinion only the channel capacity is an adequate quantity to judge the transmission quality of MIMO systems and showed, that Ray Tracing under certain circumstances produces big prediction errors when predicting the channel capacity of scenarios without a line of sight. Those errors can not be excluded in general as long as one uses Ray Tracing as prediction measure.

We then presented a new algorithm based upon the concept of Multi Channel Coupling, which accounts for an infinite number of interactions of the rays with the surrounding and thereby avoids the identified error source. The new algorithm was validated against a Ray Tracing based algorithm and against measurements.

For scenarios with line of sight, the prediction results using our approach were as good as with Image Ray Tracing, for scenarios without a line of sight, our approach outperformed the Ray Tracing based algorithm remarkably.

\section{References}

Bauch, G. and Al-Dhahir, N.: Reduced-Complexity Space-Time Turbo-Equalization for Frequency-Selective MIMO Channels, IEEE Transactions on Wireless Communications, 1, 819-829, 2002.

Elnaggar, M. S., Safavi-Naeini, S., and Chaudhuri, S. K.: Simulation of the Achievable MIMO Capacity by Using Adaptive Phased-Array, in: Proc. of the IEEE Radio and Wireless Conference (RAWCON), pp. 155-158, Atlanta (GA), 2004.

Foschini, G. J. and Gans, M. J.: On Limits of Wireless Comunication in a Fading Environment when Using Multiple Antennas, Wireless Personal Communications, 6, 311-335, 1998.

Fügen, T., Sommerkorn, G., Maurer, J., Hampicke, D., Wiesbeck, W., and Thomä, R.: MIMO Capacities for Different Antenna Array Structures Based on Double Directional Wide-Band Channel
Measurements, in: Proc. of the 13th IEEE International Symposium on Personal, Indoor and Mobile Radio Communications (PIMRC), 3, 1777-1781, Lissabon, 2002.

Hagebölling, F., Weikert, O., and Zölzer, U.: Deterministic Prediction of the Channel Capacity of Frequency Selectice MIMO Systems, in: Proc. 11th International OFDM-Workshop 2006, pp. 208-212, Hamburg, 2006.

Karthaus, U.: Vermessung und Simulation breitbandiger zeitvarianter Funkkanäle im Bereich 30-60 GHz, Dissertation, Universität Gesamthochschule Paderborn, Shaker Verlag, Aachen, ISBN 3-8265-8699-9, 2001.

Layer, F.: Synthese und Analyse zeitvarianter IndoorMobilfunkkanäle mittels strahlenoptischer Verfahren, Dissertation, Universität Gesamthochschule Kassel, Shaker Verlag, Aachen, ISBN 3-8265-8455-4, 2001.

Liebendorfer, M. and Dersch, U.: Multi Channel Coupling, a new method to predict indoor propagation, in: Proc. of the 47th IEEE Vehicular Technology Conference, Phoenix, AZ, USA, 3, 14051409, 1997.

Palomar, D., Fonollosa, J., and Lagunas, M.: Capacity Results on Frequency-Selective Rayleigh MIMO Channels, in: IST Mobile Communications Summit, Galway, Irland, pp. 491-496, 2000.

Talbi, L.: Simulation of Indoor UHF Propagation Using Numerical Technique, in: Proc. of the Canadian Conference on Electrical and Computer Engineering 2001, 2, 1357-1362, Toronto, 2001.

Tang, Z. and Mohan, A. S.: Experimenal Investigation of Indoor MIMO Ricean Channel Capacity, IEEE Antennas and Wireless Propagation Letters, 4, 55-58, 2005.

Weikert, O. and Zölzer, U.: A Flexible Laboratory MIMO System Using Four Transmit and Four Receive Antennas, in: Proc. of the 10th International OFDM-Workshop 2005, Hamburg, Germany, pp. 298-302, 2005.

Ziri-Castro, K. I., Scanlon, W. G., and Evans, N. E.: Prediction of Variation in MIMO Channel Capacity for the Populated Indoor Environment Using a Radar Cross-Section-Based Pedestrian Model, IEEE Transactions on Wireless Communications, 4, 1186-1194, 2005. 